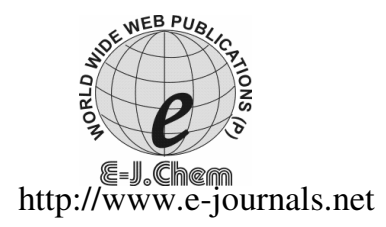

ISSN: 0973-4945; CODEN ECJHAO

E-Journal of Chemistry

2009, 6(S1), S413-S421

\title{
3-Hydroxy-2-naphthoate Complexes of Transition Metals with Hydrazine - Preparation, Spectroscopic and Thermal Studies
}

\author{
N.ARUNADEVI and S.VAIRAM* \\ Department of Chemistry, \\ Government College of Technology, Coimbatore, 641 013, India. \\ vamshen@yahoo.com
}

Received 19 June 2009; Accepted 10 August 2009

\begin{abstract}
Reaction of hydrazine and 3-hydroxy-2-naphthoic acid with some transition metal ions forms two types of complexes: (i) $\left[\mathrm{M}\left(\mathrm{N}_{2} \mathrm{H}_{4}\right)\left\{\mathrm{C}_{10} \mathrm{H}_{6}(3-\right.\right.$ $\left.\mathrm{O})(2-\mathrm{COO})\left(\mathrm{H}_{2} \mathrm{O}\right)_{2}\right]$ where $\mathrm{M}=\mathrm{Ni}, \mathrm{Co}, \mathrm{Cd}$ and $\mathrm{Zn}$, at $\mathrm{pH} 9$ and (ii) $\left[\mathrm{M}\left(\mathrm{N}_{2} \mathrm{H}_{5}\right)_{2}\left\{\mathrm{C}_{10} \mathrm{H}_{6}(3-\mathrm{O})(2-\mathrm{COO})\right\}_{2}\right] \cdot \mathrm{xH}_{2} \mathrm{O}$ where $\mathrm{M}=\mathrm{Ni} \& \mathrm{x}=1 ; \mathrm{M}=\mathrm{Co}, \mathrm{Cd}$, $\mathrm{Mn} \& \mathrm{x}=3$; and $\mathrm{M}=\mathrm{Zn}, \mathrm{Cu} \& \mathrm{x}=0$ at $\mathrm{pH} 4$. Analytical data confirms the compositions of the complexes. The acid shows dianionic nature in these complexes. The magnetic moments and electronic spectra suggest the geometry of the complexes. IR data indicates the nature of hydrazine and presence of water in the complexes. Simultaneous TG-DTA studies shows different thermal degradation patterns for the two types of complexes. The first type shows formation of no stable intermediates whereas the second type shows the respective metal hydroxy naphthoate intermediates. The final products in both the types are found to be metal oxides of nano size. XRD patterns show isomorphism among the complexes with similar molecular formulae.
\end{abstract}

Keywords: Hydrazine complexes, Hydrazinium complexes, 3-Hydroxy-2-naphthoic acid, IR, Thermal analysis.

\section{Introduction}

Hydrazine complexes of the first row transition metal ions with a variety of carboxylic acids have been reported in the literature. These include simple aliphatic mono and dicarboxylic $\operatorname{acids}^{1,2}$, aromatic mono and $d i$ carboxylic acids ${ }^{3,4}$ and heterocyclic carboxylic acids ${ }^{5}$. The hydrazinium metal carboxylates ${ }^{6}$ and their hydrates are crystalline solids and mostly water soluble but hydrazine complexes are amorphous powder and are insoluble in water. Many bis-hydrazine and hydrazinium metal carboxylates are used as precursors for metal oxides and mixed metal oxides such as ferrites, cobaltites, chromites etc ${ }^{7,8}$. 
3-Hydroxy-2-naphthoic acid is one example of a naphthalene derivative that has been frequently used as the chelating agent. Bin Liu et al. prepared $\mathrm{Cr}$ (III) complex with this chelating agent ${ }^{9}$. Fluorescence property and stability constant have been studied ${ }^{10}$. In this paper we report the preparation, characterization and thermal decomposition of some new hydrazine and hydrazinium complexes with this acid.

\section{Experimental}

The solvents were distilled prior to use and double distilled water was used for the preparation and chemical analyses. All the chemicals used were of AR grade received from Fluka Chemicals. The hydrazine hydrate was used as such as received.

Preparation of $\left[\mathrm{M}\left(\mathrm{N}_{2} \mathrm{H}_{4}\right)\left\{\mathrm{C}_{10} \mathrm{H}_{6}(3-\mathrm{O})(2-\mathrm{COO})\right\}\left(\mathrm{H}_{2} \mathrm{O}\right)_{2}\right]$ where $\mathrm{M}=\mathrm{Ni}, \mathrm{Co}, \mathrm{Cd} \& \mathrm{Zn}$

These complexes were prepared by stirring the aqueous solution of the ligands $(60 \mathrm{~mL})$ containing 3-hydroxy-2-naphthoic acid $(0.188 \mathrm{~g}, 1 \mathrm{mmol})$ and hydrazine hydrate $(0.5 \mathrm{~mL}, 10 \mathrm{mmol})$ with $20 \mathrm{~mL}$ of added metal nitrate solution (i.e.0.291 $\mathrm{g}$ of $\mathrm{Co}\left(\mathrm{NO}_{3}\right)_{2} \cdot 6 \mathrm{H}_{2} 0,1 \mathrm{mmol}$ in $20 \mathrm{~mL}$ of distilled water) at $\mathrm{pH} 9$.

The above solution mixtures which appeared cloudy at first, turned out to be a micro crystalline solid on digestion when kept over a hot water bath at $90{ }^{\circ} \mathrm{C}$ for 20 minutes. It was cooled, filtered and washed with distilled water, ethanol and then with ether. The crystals were dried in a desiccator over anhydrous $\mathrm{CaCl}_{2}$.

Preparation of $\left[\mathrm{M}\left(\mathrm{N}_{2} \mathrm{H}_{5}\right)_{2}\left\{\mathrm{C}_{10} \mathrm{H}_{6}(3-\mathrm{O})(2-\mathrm{COO})\right\}_{2}\right] \cdot \mathrm{xH}_{2} \mathrm{O}$, where $\mathrm{M}=\mathrm{Ni}, \quad x=1$; $M=C o, C d \& M n, x=3$ and $M=Z n \& C u, x=0$

A similar procedure was adopted for the preparation of these complexes, with a molar ratio of acid: base: metal ion $=1: 2: 2$, in $60 \mathrm{~mL}$ of water-alcohol $(1: 1)$ mixture at $60{ }^{\circ} \mathrm{C}$. Cadmium complex was formed with 1:1:4 ratio. The micro crystalline precipitate was formed immediately and the mixture was kept in water bath for $20 \mathrm{~min}$. It was cooled, filtered and washed with distilled water, ethanol and then with ether. The precipitate was dried in a desiccator over anhydrous $\mathrm{CaCl}_{2}$.

\section{Physicochemical techniques}

The compositions of the complexes were fixed by chemical analysis and confirmed by micro elemental analysis. Hydrazine content was determined by titrating against standard $\mathrm{KIO}_{3}$ $\left(0.025 \mathrm{~mol} \mathrm{~L}^{-1}\right)$ under Andrew's conditions. Metal contents were determined by titrating with EDTA (0.01 mol L'-1) after decomposing the complexes with 1:1 nitric acid ${ }^{11}$.

IR spectra of the complexes in the region $4000-400 \mathrm{~cm}^{-1}$ were recorded as $\mathrm{KBr}$ pellets using a Perkin Elmer 597 spectrophotometer. Electronic reflectance spectra for the solid state complexes were obtained using a Varian, Cary 5000 recording spectrophotometer. The magnetic susceptibility of the complexes was measured using a vibrating sample magnetometer, VSM EG\&G Model 155 at room temperature and the data were corrected for diamagnetism.

The X-ray powder patterns of the complexes were recorded using a Philips X-ray diffractometer (model PW 1050/70) employing $\mathrm{Cu}-\mathrm{K} \alpha$ radiation with a nickel filter. The simultaneous TG-DTA experiments were carried out using SDT Q600 V8.3 instrument and Stanton 781 simultaneous thermal analyzer. Thermal analyses were carried out in air at the heating rate of $10{ }^{\circ} \mathrm{C} \mathrm{min}^{-1}$ using 5 to $10 \mathrm{mg}$ of the samples. Platinum cups were employed as sample holders and alumina as reference. The temperature range was ambient to $700{ }^{\circ} \mathrm{C}$. 
The SEM images of the residual oxides were recorded using a Cambridge Scanning Electron Microscope with EDAX attachment (CF).

\section{Results and Discussion}

\section{Electronic spectra, magnetic susceptibility and ESR spectra}

A band appears in the range 19890-20350 $\mathrm{cm}^{-1}$ and is assigned to the ${ }^{4} \mathrm{~T}_{1 \mathrm{~g}}(\mathrm{P}) \rightarrow{ }^{4} \mathrm{~T}_{1 \mathrm{~g}}$ transition of typical six coordinated $\mathrm{Co}(\mathrm{II})$ hydrazine complexes. The corresponding nickel complexes show two bands in the region 10480-10790 and $17370-17690 \mathrm{~cm}^{-1}$ which are ascribable to ${ }^{3} \mathrm{~A}_{2 \mathrm{~g}} \rightarrow{ }^{2} \mathrm{~T}_{2 \mathrm{~g}},{ }^{3} \mathrm{~A}_{2 \mathrm{~g}} \rightarrow{ }^{2} \mathrm{~T}_{1 \mathrm{~g}}$ (F) transitions, respectively, of octahedral Ni(II). The magnetic moment values obtained for the nickel and cobalt complexes, 3.2 and 4.8 BM respectively, confirm the high spin octahedral structures.

The cobalt complexes of hydrazinium transition metal complexes register a band in the regions of 18300 and $20080 \mathrm{~cm}^{-1}$ due to transitions ${ }^{3} \mathrm{~T}_{1 \mathrm{~g}}(\mathrm{~F}) \rightarrow{ }^{4} \mathrm{~A}_{2 \mathrm{~g}}(\mathrm{~F})$ and ${ }^{3} \mathrm{~T}_{1 \mathrm{~g}}(\mathrm{~F}) \rightarrow^{4} \mathrm{~T}_{1 \mathrm{~g}}$ (P) of typical six coordinated $\mathrm{Co}(\mathrm{II})$ complexes. The corresponding nickel complexes show bands in the regions 16200,18210 and $26370 \mathrm{~cm}^{-1}$ which are ascribable to transition ${ }^{3} \mathrm{~A}_{2 \mathrm{~g}}$ $\rightarrow{ }^{3} \mathrm{~T}_{1 \mathrm{~g}}$ (F) and the copper complex shows bands in the regions 13610 and $18620 \mathrm{~cm}^{-1}$ assigned to ${ }^{2} \mathrm{~B}_{1 \mathrm{~g}} \rightarrow{ }^{2} \mathrm{~A}_{2 \mathrm{~g}}$ and ${ }^{2} \mathrm{~B}_{1 \mathrm{~g}} \rightarrow{ }^{2} \mathrm{~B}_{2 \mathrm{~g}}$ transitions, respectively, evidence the octahedral geometry of the complexes ${ }^{12}$.The magnetic moment values obtained for the nickel, cobalt, copper and manganous complexes, 3.35, 4.97, 1.83 and 5.8 BM respectively, confirm the high spin octahedral structures.

The axial ESR spectrum for copper complex shown in Figure 1 has features at $g_{\|}=2.1$ and $g_{\perp}=1.9$ which indicate that the copper(II) ion geometry is that of a distorted octahedron.

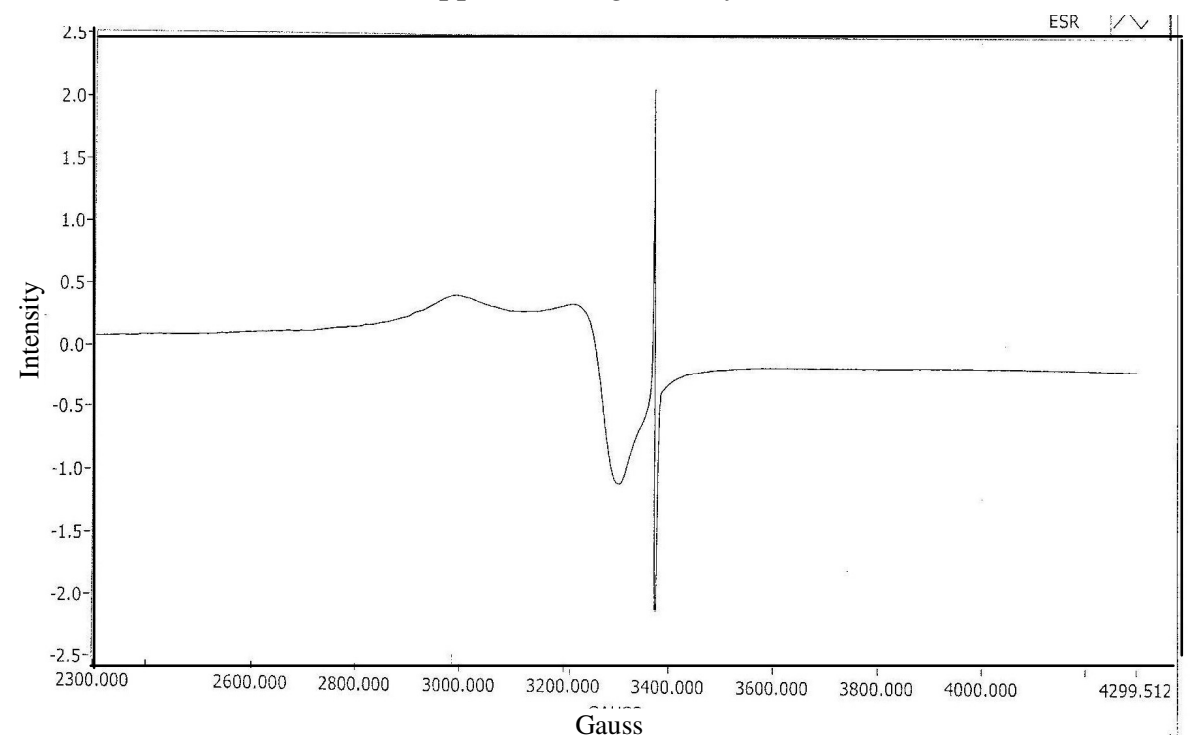

Figure 1. ESR spectrum of $\left[\mathrm{Cu}\left(\mathrm{N}_{2} \mathrm{H}_{5}\right)_{2}\left\{\mathrm{C}_{10} \mathrm{H}_{6}(3-\mathrm{O})(2-\mathrm{COO})_{2}\right\}\right]$.

\section{IR spectra of complexes}

The IR spectral data of the complexes are summarized in Table 1. 
Table 1. Analytical and IR data of the complexes.

\begin{tabular}{|c|c|c|c|c|c|c|c|c|c|c|c|c|c|}
\hline \multirow[b]{2}{*}{$\begin{array}{c}\text { Molecular Formula } \\
\text { of } \\
\text { Complexes }\end{array}$} & \multirow[b]{2}{*}{ Colour } & \multirow[b]{2}{*}{$\begin{array}{l}\text { D.pt } \\
/ \\
{ }^{\circ} \mathrm{C}\end{array}$} & \multicolumn{3}{|c|}{ Analytical data, \% } & \multicolumn{8}{|c|}{ IR data } \\
\hline & & & $\begin{array}{l}\text { C } \\
\text { Found. } \\
\text { (calcd.) }\end{array}$ & $\begin{array}{l}\mathrm{H} \quad \mathrm{N} \\
\text { Found Found } \\
\text { (calcd. (calcd. }\end{array}$ & $\begin{array}{l}\text { Hydra } \\
\text { zine Metal } \\
\text { Found. Found. } \\
\text { (calcd.) }\end{array}$ & $\begin{array}{l}\mathrm{vOH} \\
\mathrm{cm}^{-1}\end{array}$ & $\begin{array}{c}\rho_{\mathrm{r}}\left(\mathrm{H}_{2} \mathrm{O}\right) \\
\mathrm{cm}^{-1}\end{array}$ & $\begin{array}{c}\mathrm{v}_{\mathrm{C}=\mathrm{O}} \\
\text { asymm } \\
\mathrm{cm}^{-1}\end{array}$ & $\begin{array}{l}v_{\mathrm{C}=0} \\
\mathrm{sym} \\
\mathrm{cm}^{-1}\end{array}$ & $\begin{array}{l}v_{\text {asy }} \\
v_{\text {sy }} \\
\mathrm{cm}^{-1}\end{array}$ & $\begin{array}{l}v_{\mathrm{M}-\mathrm{O}} \\
\mathrm{cm}^{-1}\end{array}$ & $\begin{array}{l}v_{\mathrm{N}-\mathrm{N}} \\
\mathrm{cm}^{-1}\end{array}$ & $\begin{array}{l}v_{\mathrm{NH}} \\
\mathrm{cm}^{-1}\end{array}$ \\
\hline $\begin{array}{c}{\left[\mathrm{Ni}\left(\mathrm{N}_{2} \mathrm{H}_{4}\right)\left\{\mathrm{C}_{10} \mathrm{H}_{6}(3-\mathrm{O})\right.\right.} \\
\left.(2-\mathrm{COO})\}\left(\mathrm{H}_{2} \mathrm{O}\right)_{2}\right]\end{array}$ & Green & 135 & $\begin{array}{c}41.9 \\
(42.2)\end{array}$ & $\begin{array}{cc}4.5 & 8.7 \\
(4.5) & (8.9)\end{array}$ & $\begin{array}{cc}10.0 & 18.6 \\
(10.2) & (18.8)\end{array}$ & 3384(b) & $838(\mathrm{~s})$ & $1627(\mathrm{~m})$ & $1382(\mathrm{~m})$ & 245 & $474(s)$ & 981(s) & $3180(s)$ \\
\hline $\begin{array}{c}{\left[\mathrm{Co}\left(\mathrm{N}_{2} \mathrm{H}_{4}\right)\left\{\mathrm{C}_{10} \mathrm{H}_{6}(3-\mathrm{O})\right.\right.} \\
\left.(2-\mathrm{COO})\}\left(\mathrm{H}_{2} \mathrm{O}\right)_{2}\right]\end{array}$ & Pink & 170 & $\begin{array}{c}42.1 \\
(42.2)\end{array}$ & $\begin{array}{cc}4.4 & 8.8 \\
(4.5) & (8.9)\end{array}$ & $\begin{array}{cc}10.1 & 18.7 \\
(10.2) & (18.8)\end{array}$ & 3384(b) & $841(s)$ & 1654(s) & 1382(s) & 272 & $470(\mathrm{~s})$ & 972(s) & $3298(\mathrm{~s})$ \\
\hline $\begin{array}{c}{\left[\mathrm{Cd}\left(\mathrm{N}_{2} \mathrm{H}_{4}\right)\left\{\mathrm{C}_{10} \mathrm{H}_{6}(3-\mathrm{O})\right.\right.} \\
\left.(2-\mathrm{COO})\}\left(\mathrm{H}_{2} \mathrm{O}\right)_{2}\right]\end{array}$ & White & 180 & $\begin{array}{c}36.2 \\
(36.0)\end{array}$ & $\begin{array}{cc}3.7 & 7.5 \\
(3.8) & (7.6)\end{array}$ & $\begin{array}{cc}8.8 & 30.6 \\
(8.7) & (30.7)\end{array}$ & $3325(b)$ & $841(s)$ & $1647(\mathrm{~s})$ & $1375(\mathrm{~s})$ & 272 & $474(s)$ & $960(s)$ & $3288(\mathrm{~s})$ \\
\hline $\begin{array}{c}{\left[\mathrm{Zn}\left(\mathrm{N}_{2} \mathrm{H}_{4}\right)\left\{\mathrm{C}_{10} \mathrm{H}_{6}(3-\mathrm{O})\right.\right.} \\
\left.(2-\mathrm{COO})\}\left(\mathrm{H}_{2} \mathrm{O}\right)_{2}\right]\end{array}$ & White & 195 & $\begin{array}{c}41.3 \\
(41.3)\end{array}$ & $\begin{array}{cc}4.3 & 8.7 \\
(4.4) & (8.8)\end{array}$ & $\begin{array}{cc}10.2 & 20.5 \\
(10.0) & (20.5)\end{array}$ & 3384(b) & $840(s)$ & 1649(s) & 1394(s) & 255 & $470(\mathrm{~s})$ & $977(\mathrm{~s})$ & 3292(s) \\
\hline $\begin{array}{c}{\left[\mathrm{Ni}\left(\mathrm{N}_{2} \mathrm{H}_{5}\right)_{2}\left\{\mathrm{C}_{10} \mathrm{H}_{6}(3-\mathrm{O})\right.\right.} \\
\left.(2-\mathrm{COO})\}_{2}\right] \cdot \mathrm{H}_{2} \mathrm{O}\end{array}$ & Green & 140 & $\begin{array}{c}51.3 \\
(51.3)\end{array}$ & $\begin{array}{cc}4.6 & 10.9 \\
(4.7) & (10.9)\end{array}$ & $\begin{array}{cc}12.4 & 11.4 \\
(12.4) & (11.4)\end{array}$ & & $517(\mathrm{~s})$ & 1618(m) & $1348(b)$ & 270 & $466(s)$ & $1016(s)$ & $3299(\mathrm{~s})$ \\
\hline $\begin{array}{c}{\left[\mathrm{Co}\left(\mathrm{N}_{2} \mathrm{H}_{5}\right)_{2}\left\{\mathrm{C}_{10} \mathrm{H}_{6}(3-\mathrm{O})\right.\right.} \\
\left.(2-\mathrm{COO})\}_{2}\right] \cdot 3 \mathrm{H}_{2} \mathrm{O}\end{array}$ & Pink & 65 & $\begin{array}{c}47.9 \\
(47.9)\end{array}$ & $\begin{array}{cc}5.1 & 10.1 \\
(5.1) & (10.2)\end{array}$ & $\begin{array}{cc}11.6 & 10.7 \\
(11.6) & (10.7)\end{array}$ & 3398(b) & $580(\mathrm{~s})$ & 1604(s) & 1334(m) & 2704 & $470(\mathrm{~s})$ & $1016(s)$ & $3298(\mathrm{~s})$ \\
\hline $\begin{array}{c}{\left[\mathrm{Cd}\left(\mathrm{N}_{2} \mathrm{H}_{5}\right)_{2}\left\{\mathrm{C}_{10} \mathrm{H}_{6}(3-\mathrm{O})\right.\right.} \\
(2-\mathrm{COO})\}_{2} .3 \mathrm{H}_{2} \mathrm{O}\end{array}$ & White & 60 & $\begin{array}{c}43.7 \\
(43.7)\end{array}$ & $\begin{array}{cc}4.7 & 9.2 \\
(4.6) & (9.3)\end{array}$ & $\begin{array}{cc}10.6 & 18.5 \\
(10.6) & (18.6)\end{array}$ & 3386(b) & $573(\mathrm{~s})$ & $1600(\mathrm{~m})$ & $1326(\mathrm{~m})$ & 274 & $470(s)$ & $1016(\mathrm{~s})$ & $3282(\mathrm{~s})$ \\
\hline $\begin{array}{c}{\left[\mathrm{Zn}\left(\mathrm{N}_{2} \mathrm{H}_{5}\right)_{2}\left\{\mathrm{C}_{10} \mathrm{H}_{6}(3-\mathrm{O})\right.\right.} \\
\left.(2-\mathrm{COO})\}_{2}\right]\end{array}$ & $\begin{array}{l}\text { Dull } \\
\text { white }\end{array}$ & 280 & $\begin{array}{c}52.5 \\
(52.4)\end{array}$ & $\begin{array}{cc}4.3 & 11.1 \\
(4.4) & (11.1)\end{array}$ & $\begin{array}{cc}12.7 & 12.9 \\
(12.7) & (13.0)\end{array}$ & - & $596(\mathrm{~s})$ & $1649(\mathrm{~s})$ & $1363(\mathrm{~m})$ & 2864 & $476(s)$ & $1014(\mathrm{~s})$ & $3265(s)$ \\
\hline $\begin{array}{c}{\left[\mathrm{Mn}\left(\mathrm{N}_{2} \mathrm{H}_{5}\right)_{2}\left\{\mathrm{C}_{10} \mathrm{H}_{6}(3-\mathrm{O})\right.\right.} \\
\left.(2-\mathrm{COO})\}_{4}\right] .3 \mathrm{H}_{2} \mathrm{O}\end{array}$ & $\begin{array}{l}\text { Dull } \\
\text { white }\end{array}$ & 130 & $\begin{array}{c}48.3 \\
(48.3)\end{array}$ & $\begin{array}{cc}5.1 & 10.2 \\
(5.1) & (10.2)\end{array}$ & $\begin{array}{cc}11.7 & 10.0 \\
(11.7) & (10.0)\end{array}$ & 3346(b) & $596(\mathrm{~s})$ & 1649(s) & $1363(\mathrm{~m})$ & 2864 & $470(\mathrm{~s})$ & $1014(\mathrm{~s})$ & $3265(s)$ \\
\hline $\begin{array}{c}{\left[\mathrm{Cu}\left(\mathrm{N}_{2} \mathrm{H}_{5}\right)_{2}\left\{\mathrm{C}_{10} \mathrm{H}_{6}(3-\mathrm{O})\right.\right.} \\
\left.\left.(2-\mathrm{COO})_{2}\right\}\right]\end{array}$ & $\begin{array}{l}\text { Grass } \\
\text { green }\end{array}$ & 175 & $\begin{array}{c}52.6 \\
(52.6)\end{array}$ & $\begin{array}{cc}4.4 & 11.2 \\
(4.4) & (11.2)\end{array}$ & $\begin{array}{cc}12.8 & 12.6 \\
(12.8) & (12.7)\end{array}$ & - & $594(s)$ & $1647(\mathrm{~m})$ & $1396(\mathrm{~m})$ & 251 & $472(s)$ & $1014(\mathrm{~s})$ & $3286(s)$ \\
\hline
\end{tabular}


All the hydrated complexes, except zinc and copper in hydrazinium complexes, display a medium broad peak centered on $3440 \mathrm{~cm}^{-1}$, indicating the $\mathrm{O}-\mathrm{H}$ stretch of water molecules. An additional sharp peak in the region $838-841 \mathrm{~cm}^{-1}$ is observed which may be due to the presence of coordinated water molecules in $\left[\mathrm{M}\left(\mathrm{N}_{2} \mathrm{H}_{4}\right)\left\{\mathrm{C}_{10} \mathrm{H}_{6}(3-\mathrm{O})(2-\mathrm{COO})\right\}\left(\mathrm{H}_{2} \mathrm{O}\right)_{2}\right]$ and sharp peak in the region $517-596 \mathrm{~cm}^{-1}$ is due to presence of lattice water in $\left[\mathrm{M}\left(\mathrm{N}_{2} \mathrm{H}_{5}\right)_{2}\left\{\mathrm{C}_{10} \mathrm{H}_{6}(3-\mathrm{O})(2-\mathrm{COO})\right\}_{2}\right] \cdot \mathrm{xH}_{2} \mathrm{O}$. Infrared technique serves as finger print technique and the presence of bidentate $\mathrm{N}_{2} \mathrm{H}_{4}$ in $\left[\mathrm{M}\left(\mathrm{N}_{2} \mathrm{H}_{4}\right)\left\{\mathrm{C}_{10} \mathrm{H}_{6}(3-\mathrm{O})(2-\mathrm{COO})\left(\mathrm{H}_{2} \mathrm{O}\right)_{2}\right]\right.$ is indicated by the characteristic $N-N$ frequency in the range $960-981 \mathrm{~cm}^{-1}$. The presence of coordinated hydrazinium cation in $\left[\mathrm{M}\left(\mathrm{N}_{2} \mathrm{H}_{5}\right)_{2}\left\{\mathrm{C}_{10} \mathrm{H}_{6}(3-\mathrm{O})(2-\mathrm{COO})\right\}_{2}\right] \cdot \mathrm{xH}_{2} \mathrm{O}$ has been indicated by the presence of $v_{\mathrm{N}-\mathrm{N}}$ in the region $1014-1016 \mathrm{~cm}^{-1}{ }^{13}$. All the compounds show moderately strong bands in the region $3180-3299 \mathrm{~cm}^{-1}$ due to $\mathrm{N}-\mathrm{H}$ stretching. The asymmetric and symmetric stretching frequencies of carboxylate ions are seen in the range $1600-1654$ and $1326-1396 \mathrm{~cm}^{-1}$, respectively with a $\left(v_{\text {asy }}-v_{\text {sy }}\right)$ separation of $245-286 \mathrm{~cm}^{-1}$ indicating the monodentate linkage of both the carboxylate group.

\section{Thermal analysis}

The thermal data of the complexes are listed in Table 2. The compositions of the intermediates and the final products are those, which fit with the observed mass losses in TG. Thermogravimetric results are in good agreement with the DTA data. As a representative example, TG-DTA of $\left[\mathrm{Ni}_{(}\left(\mathrm{N}_{2} \mathrm{H}_{4}\right)\left\{\mathrm{C}_{10} \mathrm{H}_{6}(3-\mathrm{O})(2-\mathrm{COO})\right\}\left(\mathrm{H}_{2} \mathrm{O}\right)_{2}\right]$ and $\left[\mathrm{Ni}\left(\mathrm{N}_{2} \mathrm{H}_{5}\right)_{2}\left\{\mathrm{C}_{10} \mathrm{H}_{6}(3-\mathrm{O})(2-\mathrm{COO})\right\}_{2}\right] \cdot \mathrm{H}_{2} \mathrm{O}$ are shown in Figure $2(\mathrm{a}) \& 2(\mathrm{~b})$.

Table 2 . Thermal Analysis of the complexes.

\begin{tabular}{|c|c|c|c|c|c|}
\hline \multirow[b]{2}{*}{$\begin{array}{l}\text { Molecular formula of } \\
\text { the complexes }\end{array}$} & \multirow[b]{2}{*}{$\begin{array}{l}\text { DTA peak } \\
\text { Temp, }{ }^{\circ} \mathrm{C}\end{array}$} & \multicolumn{3}{|c|}{ TG } & \multirow[b]{2}{*}{ Decomposition products } \\
\hline & & $\begin{array}{c}\text { Temp. } \\
\text { Range, }{ }^{\circ} \mathrm{C}\end{array}$ & $\frac{\text { Weight }}{\text { obsd. }}$ & $\frac{\text { loss, } \%}{\text { calcd. }}$ & \\
\hline \multirow{2}{*}{$\begin{array}{c}\mathrm{Ni}\left(\mathrm{N}_{2} \mathrm{H}_{4}\right)\left\{\mathrm{C}_{10} \mathrm{H}_{6}(3-\mathrm{O})\right. \\
\left.(2-\mathrm{COO})\}\left(\mathrm{H}_{2} \mathrm{O}\right)_{2}\right]\end{array}$} & $140(+) 223(-)$ & $40-259$ & 21.3 & - & \multirow{2}{*}{$\begin{array}{l}\text { unstable intermediate } \\
\mathrm{NiO}\end{array}$} \\
\hline & $352(-)$ & $259-700$ & 76.1 & 76.1 & \\
\hline \multirow{2}{*}{$\begin{array}{c}\mathrm{Co}\left(\mathrm{N}_{2} \mathrm{H}_{4}\right)\left\{\mathrm{C}_{10} \mathrm{H}_{6}(3-\mathrm{O})\right. \\
\left.(2-\mathrm{COO})\}\left(\mathrm{H}_{2} \mathrm{O}\right)_{2}\right] \\
\end{array}$} & $238(-)$ & $179-257$ & 21.3 & - & \multirow{2}{*}{$\begin{array}{c}\text { unstable intermediate } \\
\mathrm{Co}_{3} \mathrm{O}_{4}\end{array}$} \\
\hline & $365(-)$ & $257-700$ & 74 & 74.4 & \\
\hline \multirow{2}{*}{$\begin{array}{c}{\left[\mathrm{Cd}\left(\mathrm{N}_{2} \mathrm{H}_{4}\right)\left\{\mathrm{C}_{10} \mathrm{H}_{6}(3-\mathrm{O})\right.\right.} \\
\left.(2-\mathrm{COO})\}\left(\mathrm{H}_{2} \mathrm{O}\right)_{2}\right] \\
\end{array}$} & $227(-)$ & $189-244$ & 24.4 & - & \multirow{2}{*}{$\begin{array}{l}\text { unstable intermediate } \\
\text { CdO }\end{array}$} \\
\hline & $431(-)$ & $244-700$ & 64.8 & 64.9 & \\
\hline \multirow{2}{*}{$\begin{array}{c}{\left[\mathrm{Zn}\left(\mathrm{N}_{2} \mathrm{H}_{4}\right)\left\{\mathrm{C}_{10} \mathrm{H}_{6}(3-\mathrm{O})\right.\right.} \\
\left.\quad(2-\mathrm{COO})\}\left(\mathrm{H}_{2} \mathrm{O}\right)_{2}\right]\end{array}$} & $215(+)$ & $196-233$ & 11.1 & 11.3 & \multirow{2}{*}{$\begin{array}{c}{\left[\mathrm{Zn}\left(\mathrm{N}_{2} \mathrm{H}_{4}\right)\left\{\mathrm{C}_{10} \mathrm{H}_{6}\right.\right.} \\
(3-\mathrm{O})(2-\mathrm{COO})]\} \\
\text { unstable intermediate } \\
\mathrm{ZnO}\end{array}$} \\
\hline & $\begin{array}{l}267(-) \\
453(-)\end{array}$ & $\begin{array}{l}233-359 \\
359-700\end{array}$ & $\begin{array}{l}21.5 \\
74.4\end{array}$ & - & \\
\hline \multirow{2}{*}{$\begin{array}{l}{\left[\mathrm{Ni}\left(\mathrm{N}_{2} \mathrm{H}_{5}\right)_{2}\left\{\mathrm{C}_{10} \mathrm{H}_{6}(3-\mathrm{O})\right.\right.} \\
\left.\quad(2-\mathrm{COO})\}_{2}\right] \cdot \mathrm{H}_{2} \mathrm{O}\end{array}$} & $146(+)$ & $57-166$ & 3.5 & 3.5 & $\begin{array}{c}\left(\mathrm{N}_{2} \mathrm{H}_{5}\right)_{2} \mathrm{Ni}\left\{\mathrm{C}_{10} \mathrm{H}_{6}\right. \\
(3-\mathrm{O})(2-\mathrm{COO})\}_{2}\end{array}$ \\
\hline & $\begin{array}{r}296(-) 318(-) \\
498(-)\end{array}$ & $166-700$ & 85.3 & 85.5 & \multirow{3}{*}{$\begin{array}{c}\left(\mathrm{N}_{2} \mathrm{H}_{5}\right)_{2} \mathrm{Co}\left\{\mathrm{C}_{10} \mathrm{H}_{6}\right. \\
(3-\mathrm{O})(2-\mathrm{COO})\}_{2} \\
\mathrm{Co}_{3} \mathrm{O}_{4}\end{array}$} \\
\hline \multirow[b]{2}{*}{$\begin{array}{c}{\left[\mathrm{Co}\left(\mathrm{N}_{2} \mathrm{H}_{5}\right)_{2}\left\{\mathrm{C}_{10} \mathrm{H}_{6}(3-\mathrm{O})\right.\right.} \\
\left.\quad(2-\mathrm{COO})\}_{2}\right] \cdot 3 \mathrm{H}_{2} \mathrm{O}\end{array}$} & $79(+)$ & $43-100$ & 10.1 & 9.8 & \\
\hline & $\begin{array}{l}279(-) \\
429(-)\end{array}$ & $\begin{array}{c}100- \\
700\end{array}$ & 84.7 & 85.4 & \\
\hline \multirow{2}{*}{$\begin{array}{c}{\left[\mathrm{Cd}\left(\mathrm{N}_{2} \mathrm{H}_{5}\right)_{2}\left\{\mathrm{C}_{10} \mathrm{H}_{6}(3-\mathrm{O})\right.\right.} \\
\quad(2-\mathrm{COO})\}_{2} .3 \mathrm{H}_{2} \mathrm{O}\end{array}$} & $61(+)$ & $40-100$ & 8.6 & 8.9 & \multirow{2}{*}{$\begin{array}{c}\left(\mathrm{N}_{2} \mathrm{H}_{5}\right)_{2} \mathrm{Cd}\left\{\mathrm{C}_{10} \mathrm{H}_{6}\right. \\
(3-\mathrm{O})(2-\mathrm{COO})\}_{2} \\
\mathrm{CdO}\end{array}$} \\
\hline & $409(-)$ & $100-700$ & 78.6 & 78.7 & \\
\hline
\end{tabular}




\begin{tabular}{|c|c|c|c|c|c|}
\hline 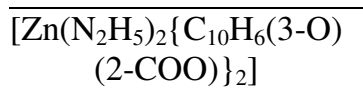 & $\begin{array}{l}282(+) \\
496(-)\end{array}$ & $\begin{array}{l}200-330 \\
330-700\end{array}$ & $\begin{array}{l}12.3 \\
83.5\end{array}$ & $\begin{array}{l}12.5 \\
83.8\end{array}$ & $\begin{array}{c}\mathrm{Zn}\left\{\mathrm{C}_{10} \mathrm{H}_{6}(3-\mathrm{O})(2-\mathrm{COO})\right\}_{2} \\
\mathrm{ZnO}\end{array}$ \\
\hline \multirow{2}{*}{$\begin{array}{c}{\left[\mathrm{Mn}\left(\mathrm{N}_{2} \mathrm{H}_{5}\right)_{2}\left\{\mathrm{C}_{10} \mathrm{H}_{6}(3-\mathrm{O})\right.\right.} \\
\left.\quad(2-\mathrm{COO})\}_{4}\right] \cdot 3 \mathrm{H}_{2} \mathrm{O}\end{array}$} & $135(+)$ & $42-200$ & 10.0 & 9.9 & \multirow{2}{*}{$\begin{array}{c}\left(\mathrm{N}_{2} \mathrm{H}_{5}\right)_{2} \mathrm{Mn}\left\{\mathrm{C}_{10} \mathrm{H}_{6}\right. \\
(3-\mathrm{O})(2-\mathrm{COO})\}_{2} \\
\mathrm{MnO}_{2}\end{array}$} \\
\hline & 424 & $200-700$ & 84.2 & 84.0 & \\
\hline \multirow{2}{*}{$\begin{array}{c}{\left[\mathrm{Cu}\left(\mathrm{N}_{2} \mathrm{H}_{5}\right)_{2}\left\{\mathrm{C}_{10} \mathrm{H}_{6}(3-\mathrm{O})\right.\right.} \\
\left.\left.\quad(2-\mathrm{COO})_{2}\right\}\right]\end{array}$} & 178 & $100-237$ & 12.6 & 12.8 & \multirow{2}{*}{$\begin{array}{c}\mathrm{Cu}\left\{\mathrm{C}_{10} \mathrm{H}_{6}(3-\mathrm{O})\right. \\
(2-\mathrm{COO})\}_{2} \\
\mathrm{CuO}\end{array}$} \\
\hline & $354(-)$ & $237-700$ & 84.1 & 84.2 & \\
\hline
\end{tabular}

$(+)=$ endotherm $;(-)=$ exotherm

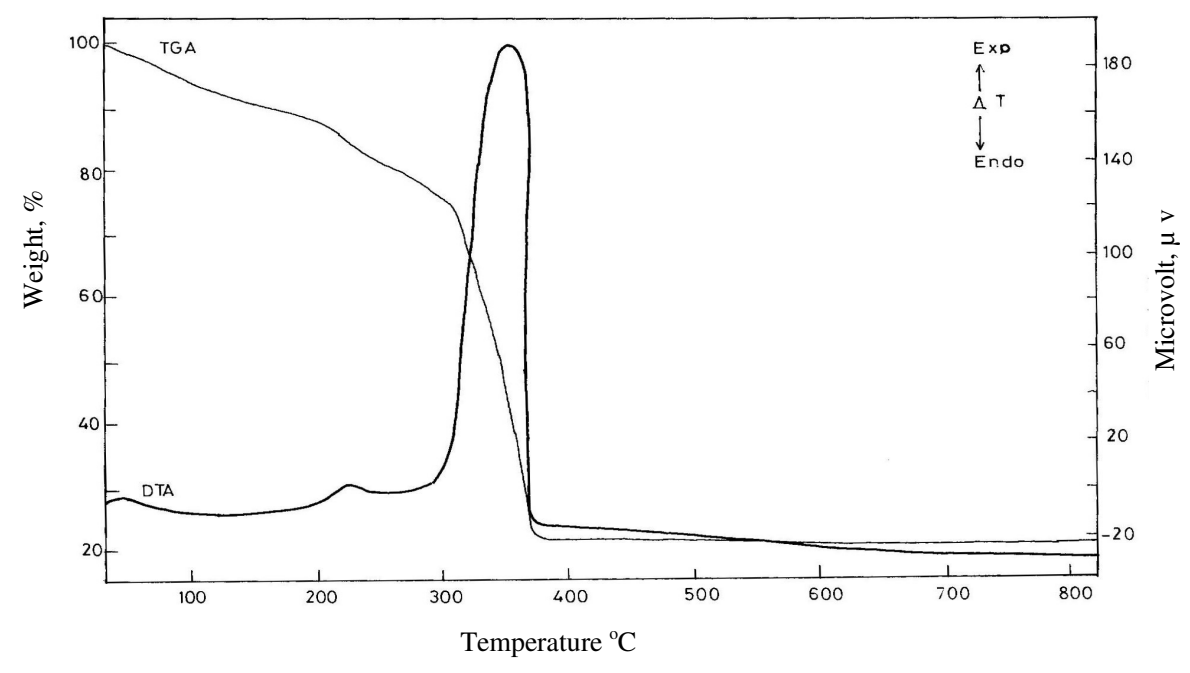

Figure 2(a). TG-DTA of $\left[\mathrm{Ni}\left(\mathrm{N}_{2} \mathrm{H}_{4}\right)\left\{\mathrm{C}_{10} \mathrm{H}_{6}(3-\mathrm{O})(2-\mathrm{COO})\right\}\left(\mathrm{H}_{2} \mathrm{O}\right)_{2}\right]$.

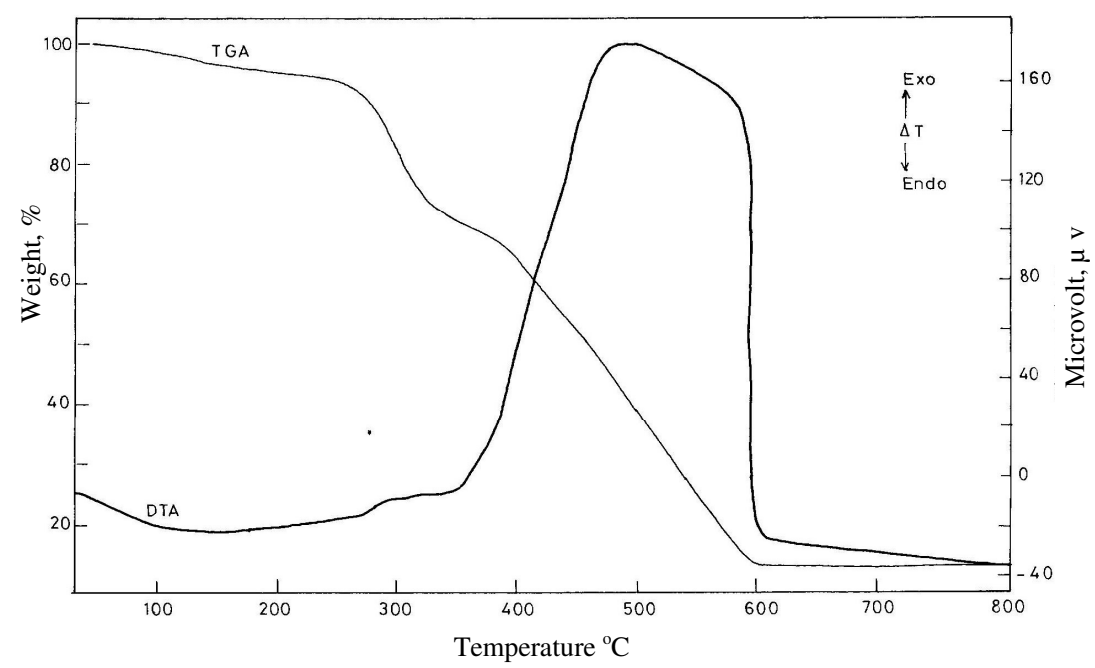

Figure 2(b). TG-DTA of $\left[\mathrm{Ni}\left(\mathrm{N}_{2} \mathrm{H}_{5}\right)_{2}\left\{\mathrm{C}_{10} \mathrm{H}_{6}(3-\mathrm{O})(2-\mathrm{COO})\right\}_{2}\right] . \mathrm{H}_{2} \mathrm{O}$ 


\section{$\left[\mathrm{M}\left(\mathrm{N}_{2} \mathrm{H}_{4}\right)\left\{\mathrm{C}_{10} \mathrm{H}_{6}(3-\mathrm{O})(2-\mathrm{COO})\right\}\left(\mathrm{H}_{2} \mathrm{O}\right)_{2}\right]$ where $\mathrm{M}=\mathrm{Ni}, \mathrm{Co}, \mathrm{Cd}$ and $\mathrm{Zn}$}

Nickel, cobalt and cadmium complexes lose hydrazine and water simultaneously showing exotherms at 223, 238 and $227{ }^{\circ} \mathrm{C}$ forming an unstable intermediate which could not be made out. Dehydration normally accompanies heat absorption. A broad endotherm centred at $140{ }^{\circ} \mathrm{C}$ due to dehydration appears initially in the case of nickel complex. But in the case of other complexes, this step appears to combine with dehydrazination resulting in exotherms in the range $227-238^{\circ} \mathrm{C}$. During the last step of decomposition of the complexes, the unstable intermediate degrades to the respective metal oxide showing a strong exothermic peak in the range $352-431{ }^{\circ} \mathrm{C}$.

A clear distinct mass loss is observed in the TG of $\mathrm{Zn}$ complex, showing endothermic dehydration at $215{ }^{\circ} \mathrm{C}$, followed by an exothermic decomposition at $267{ }^{\circ} \mathrm{C}$ with the formation of unstable intermediate. During the last step this intermediate undergoes exothermic decomposition at $453^{\circ} \mathrm{C}$ forming $\mathrm{ZnO}$ as final product.

$$
\left[\mathrm{M}\left(\mathrm{N}_{2} \mathrm{H}_{5}\right)_{2}\left\{\mathrm{C}_{10} \mathrm{H}_{6}(3-\mathrm{O})(2-\mathrm{COO})\right\}_{2}\right] . \mathrm{xH}_{2} \mathrm{O} \text {, where } \mathrm{M}=\mathrm{Ni}, \mathrm{Co}, \mathrm{Cd}, \mathrm{Mn}, \mathrm{Zn} \& \mathrm{Cu}
$$

All the complexes undergo two stages of decomposition upon heating. For $\mathrm{Ni}, \mathrm{Co}, \mathrm{Cd}$ and $\mathrm{Mn}$ complexes, the first stage of decomposition occurs in the range $61-146{ }^{\circ} \mathrm{C}$ which is attributed to the loss of water molecules present outside the co-ordination sphere. On further heating, the anhydrous complexes decomposes directly to $\mathrm{NiO}, \mathrm{Co}_{3} \mathrm{O}_{4}, \mathrm{CdO}$ and $\mathrm{MnO}_{2}$ in the temperature range $279-498{ }^{\circ} \mathrm{C}$ which was confirmed by their XRD pattern.

Zinc and copper complexes undergoes dehydrazination, forms an unstable intermediate,

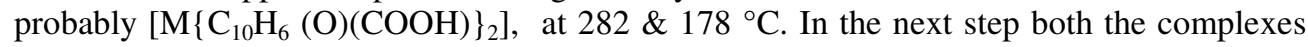
undergo exothermic decomposition to form corresponding metal oxides as final product at $496{ }^{\circ} \mathrm{C}$ and $354^{\circ} \mathrm{C}$.

The metal oxides formed after the incineration of the complexes at their decomposition points found from DTA, followed by sintering at the same temperature for about 3-4 h were found to be pure and uniform in nano scale $(20-25 \mathrm{~nm})$ as found from XRD using Scherer's formula $^{14}, \mathrm{D}=\mathrm{K} \lambda / \mathrm{B} \cos \theta$ where $\lambda$ is the $\mathrm{X}$-ray wavelength, $\mathrm{B}$ is the full width of height maximum (FWHM) of a diffraction peak, $\theta$ is the diffraction angle and $\mathrm{K}$ is the Scherrer's constant of the order of 0.8 . The SEM image of $\mathrm{MnO}_{2}$ from hydrazinium complex is shown as representative example (Figure 3 ).

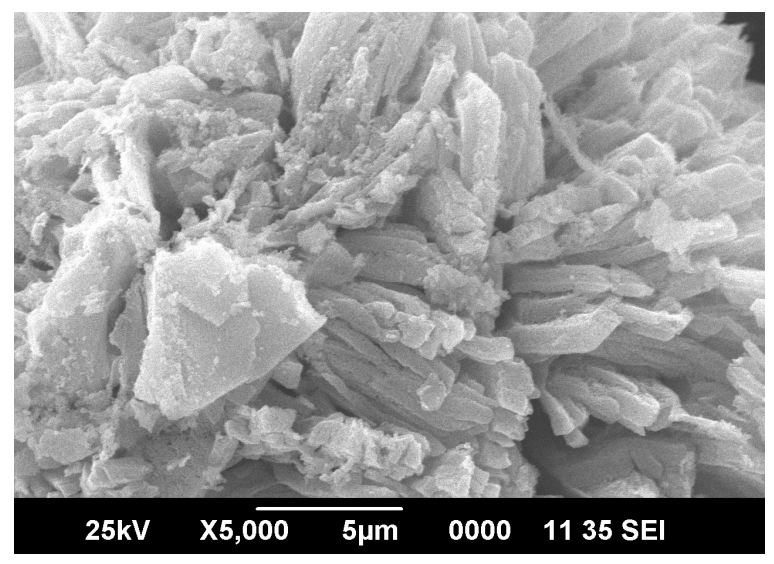

Figure 3. SEM image of $\mathrm{MnO}_{2}$. 
Table 3. $\mathrm{X}$ ray powder diffraction data of 3-hydroxy - 2-naphthoic acid complexes (d spacings in $\mathrm{A}^{\circ}$ and relative intensities in parentheses).

\begin{tabular}{|c|c|c|c|c|c|c|c|c|c|}
\hline \multicolumn{4}{|c|}{$\left[\mathrm{M}\left(\mathrm{N}_{2} \mathrm{H}_{4}\right)\left\{\mathrm{C}_{10} \mathrm{H}_{6}(3-\mathrm{O})(2-\mathrm{COO})\right\}\left(\mathrm{H}_{2} \mathrm{O}\right)_{2}\right]$} & \multirow{2}{*}{$\begin{array}{c}{\left[\mathrm{Ni}\left(\mathrm{N}_{2} \mathrm{H}_{5}\right)_{2}\right.} \\
\left\{\mathrm{C}_{10} \mathrm{H}_{6}(3-\mathrm{O})\right. \\
\left.(2-\mathrm{COO})\}_{2}\right] \cdot \mathrm{H}_{2} \mathrm{O}\end{array}$} & \multicolumn{3}{|c|}{$\begin{array}{c}{\left[\mathrm{M}\left(\mathrm{N}_{2} \mathrm{H}_{5}\right)_{2}\left\{\mathrm{C}_{10} \mathrm{H}_{6}(3-\mathrm{O})\right.\right.} \\
\left.\quad(2-\mathrm{COO})\}_{2}\right] \cdot 3 \mathrm{H}_{2} \mathrm{O}\end{array}$} & \multicolumn{2}{|c|}{$\begin{array}{c}{\left[\mathrm{M}\left(\mathrm{N}_{2} \mathrm{H}_{5}\right)_{2}\left\{\mathrm{C}_{10} \mathrm{H}_{6}\right.\right.} \\
\left.(3-\mathrm{O})(2-\mathrm{COO})\}_{2}\right]\end{array}$} \\
\hline $\mathrm{Ni}$ & $\mathrm{Co}$ & $\mathrm{Cd}$ & $\mathrm{Zn}$ & & $\mathrm{Co}$ & $\mathrm{Cd}$ & $\mathrm{Mn}$ & $\mathrm{Zn}$ & $\mathrm{Cu}$ \\
\hline $11.7505(28.8)$ & $11.6308(14.4)$ & $11.6382(8.3)$ & $11.5048(13.8)$ & $19.52(65.9)$ & $17.01(12)$ & $17.14(100)$ & $17.15(45.9)$ & $17.57(100)$ & $18.48(100)$ \\
\hline $10.3741(24.2)$ & $10.4457(24.1)$ & $10.3094(7.5)$ & $10.3069(15.8)$ & $4.7821(95.3)$ & $15.66(16)$ & $15.55(8.4)$ & $15.74(100)$ & $6.31(12.3)$ & $6.32(68)$ \\
\hline $9.28272(29.1)$ & $9.3959(33)$ & $9.3429(6.5)$ & $9.0835(9.4)$ & 4.0107 (99.7) & $5.9737(100)$ & $5.86(14.1)$ & $5.33(59.4)$ & $5.06(28.3)$ & $5.09(72.9)$ \\
\hline $6.0387(100)$ & $5.9346(78.5)$ & $6.4373(100)$ & $5.9402(100)$ & $3.8499(89.5)$ & $3.6045(74.4)$ & $3.16(21.3)$ & $3.89(92.9)$ & $4.54(18.5)$ & $4.55(75.5)$ \\
\hline $4.4285(67.3)$ & $4.4601(78.3)$ & $4.4828(8.1)$ & $4.4856(16.1)$ & $3.3456(89.6)$ & $2.7845(70.5)$ & 2.68 & $2.67(98.1)$ & $3.65(25.3)$ & $3.63(80.9)$ \\
\hline $3.9974(89.8)$ & $3.7084(100)$ & $3.6301(10.1)$ & $3.6664(25.6)$ & $2.0471(63.1)$ & $2.1104(59.2)$ & 2.28 & $2.28(93.8)$ & $2.10(5.3)$ & $2.22(57.3)$ \\
\hline \multirow[t]{6}{*}{$2.8967(70.1)$} & $2.8126(85)$ & $2.7999(5.4)$ & $2.8192(20.7)$ & $1.9974(61.7)$ & $1.8303(57.1)$ & $1.93(5.9)$ & $1.92(92)$ & $1.57(3.3)$ & $1.57(45.3)$ \\
\hline & & & & $1.9512(63.4)$ & $1.5988(51.2)$ & $1.58(6.6)$ & $1.58(89.5)$ & & \\
\hline & & & & $1.3345(54)$ & & & & & \\
\hline & & & & $1.46(48.1)$ & & & & & \\
\hline & & & & $1.3006(35.3)$ & & & & & \\
\hline & & & & $1.2584(34.5)$ & & & & & \\
\hline
\end{tabular}




\section{$X$-ray diffraction}

$\mathrm{X}$-ray powder diffraction data of the complexes are summarized in Table 3. It is found that the diffraction patterns of hydrazine complexes of $\mathrm{Ni}, \mathrm{Co}, \mathrm{Cd} \& \mathrm{Zn}$, hydrazinium complexes of $\mathrm{Co}, \mathrm{Cd} \& \mathrm{Mn}$ and hydrazinium complexes of $\mathrm{Zn} \& \mathrm{Cu}$ show similarity among the individuals in each set implying isomorphism

\section{Conclusion}

Transition metal ions such as $\mathrm{Ni}(\mathrm{II}), \mathrm{Co}(\mathrm{II}), \mathrm{Cd}(\mathrm{II}), \mathrm{Mn}(\mathrm{II}), \mathrm{Zn}(\mathrm{II}) \& \mathrm{Cu}(\mathrm{II})$ react with 3-hydroxy-2-naphthoic acid and hydrazine hydrate yield hydrazine metal naphthoates at $\mathrm{pH}$ 9 and hydrazinium metal naphthoates at $\mathrm{pH} 4$. The magnetic moment and electronic spectra suggest the high-spin octahedral nature of the complexes. Infrared spectra indicate the monodentate nature of carboxylate ions, bidentate bridged nature of hydrazine in bis-hydrazine complexes and coordinated hydrazinium cation in hydrazinium complexes. TG-DTA studies shows different thermal degradation patterns for the two types of complexes. The first type shows formation of no stable intermediates whereas the second type shows the respective metal hydroxy naphthoate intermediates. During the last step of decomposition all the complexes undergo oxidative decomposition to form corresponding metal oxides as final product. Because of the evolution of various gases during decomposition $\left(\mathrm{N}_{2}, \mathrm{H}_{2}\right.$ and $\left.\mathrm{CO}_{2}\right)$, the metal oxides formed are fine particles probably with nano size $20-25 \mathrm{~nm}$.

\section{References}

1. Sivasankar B N and Govindrajan S, J Therm Anal., 1997, 48, 1401.

2. Yasodhai S and Govindrajan S, Synth React Inorg Met Org Chem., 2000, 30, 745.

3. Kuppusamy K and Govindrajan S, Synth React Inorg Met Org Chem., 1996, 26, 225.

4. Kuppusamy K and Govindrajan S, Eur J Solid State Inorg Chem., 1995, 32, 997.

5. Premkumar T and Govindrajan S, Thermochim Acta, 2002, 35, 386.

6. Vairam S and Govindarajan S, Polish J Chem., 2006, 80, 1601.

7. Ravindranathan P and Patil K C, Amer Ceram Soc Bull., 1987, 66, 688.

8. Ravindranathan P, Mahesh G V and Patil K C, J Solid State Chem., 1987,66, 20.

9. Bin Liu, Ying-qi Li and Bin-Sheng Yang, Inorg Chem Comm., 2007, 10, 367.

10. Rodriguez-Caceres M I, Agharia R A and Warner I M, J Fluorescence, 2005, 15, 185.

11. Vogel A I, A textbook of quantitative inorganic analysis including elementary instrumental analysis, ${ }^{\text {rd }}$ Ed., Longmans Green, London, 1961.

12. Lever A B P, Inorganic Electronic Spectroscopy, Elsevier, Amsterdam, 1984.

13. Braibanti A, Dallavalle F, Pellinghelli M A and Laporati E, Inorg Chem.,1968, 7, 1430.

14. Guozhong Cao, Nano Structures and Nano materials, Synthesis, Properties and applications, Imperial College Press, London, 2004. 


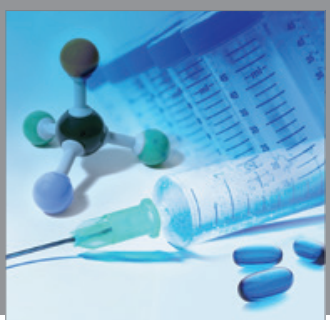

International Journal of

Medicinal Chemistry

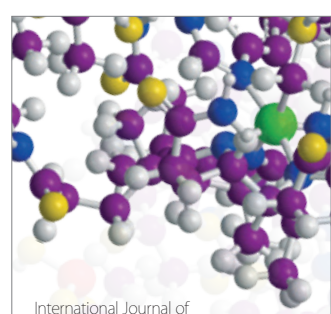

Carbohydrate Chemistry

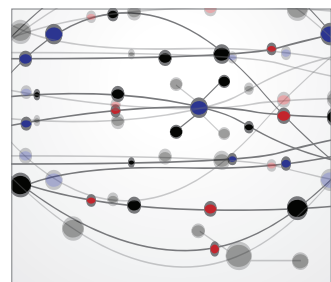

The Scientific World Journal
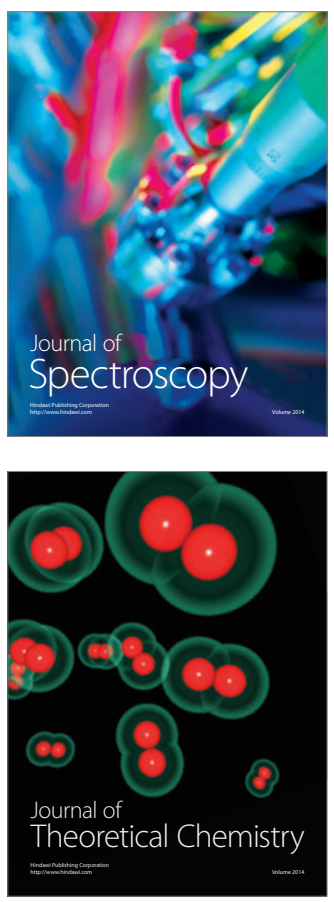
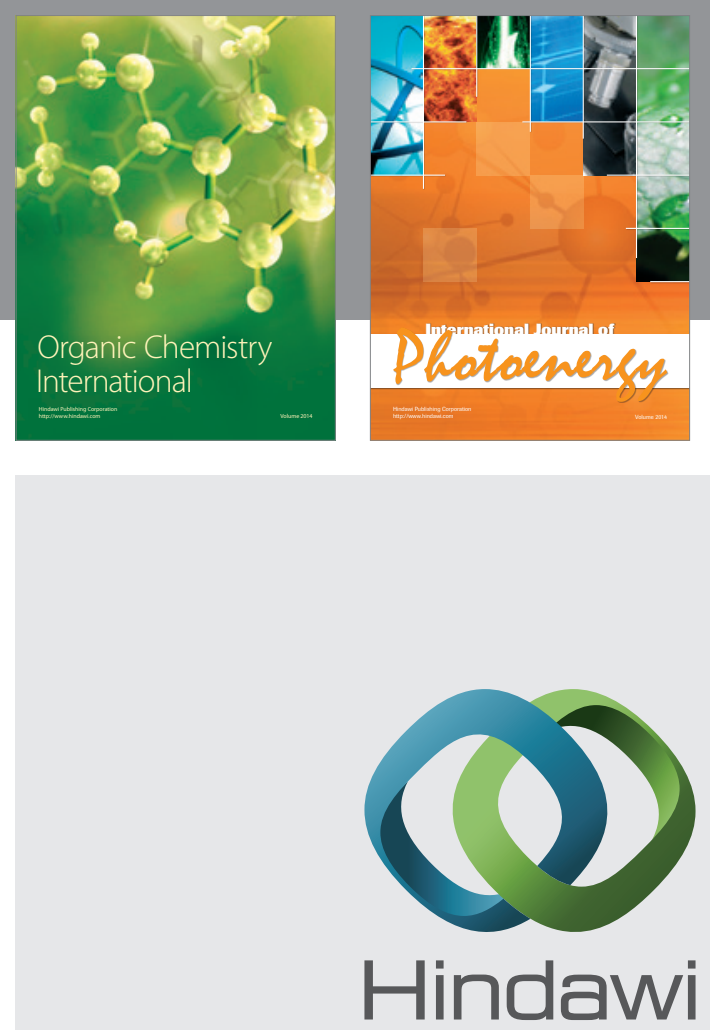

Submit your manuscripts at

http://www.hindawi.com
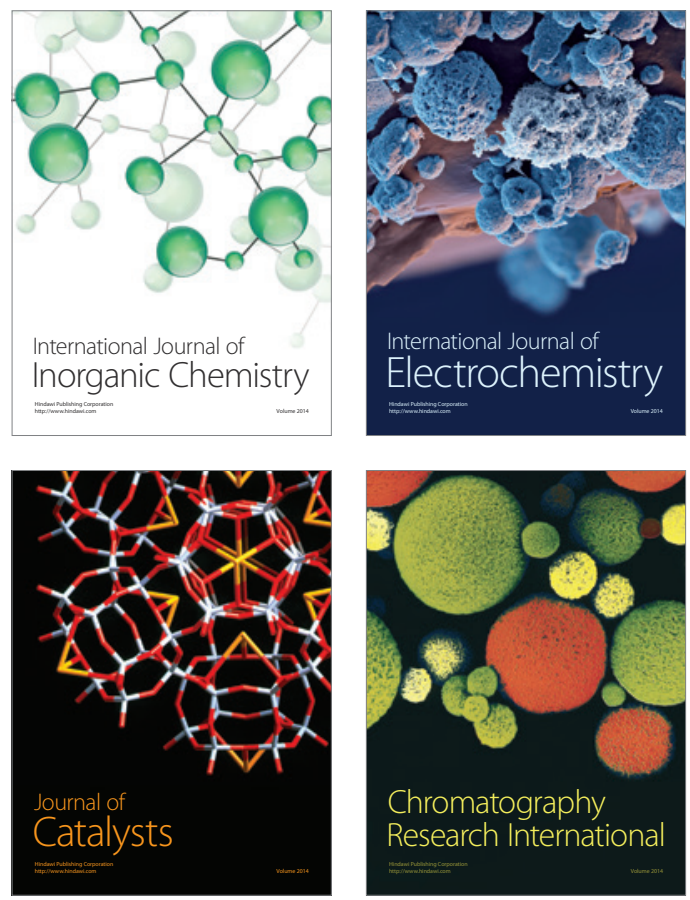
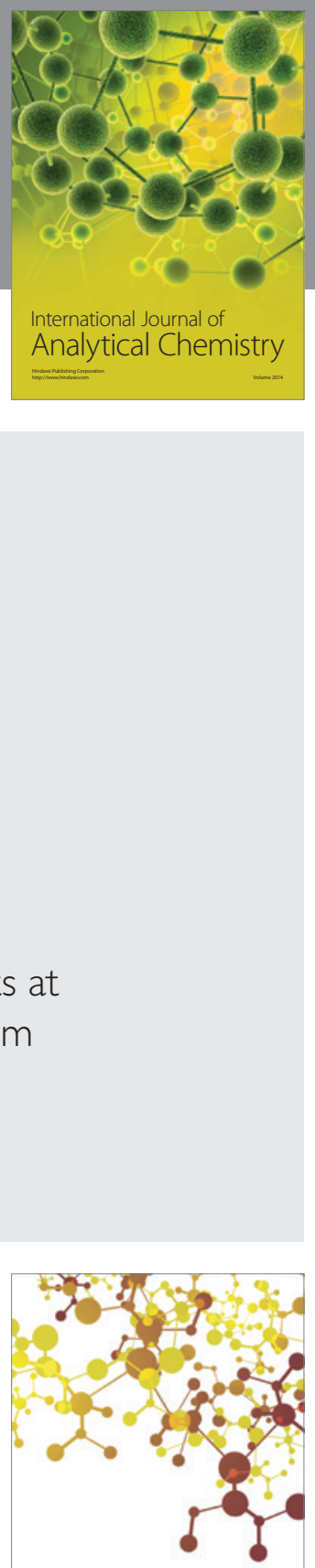

Journal of

Applied Chemistry
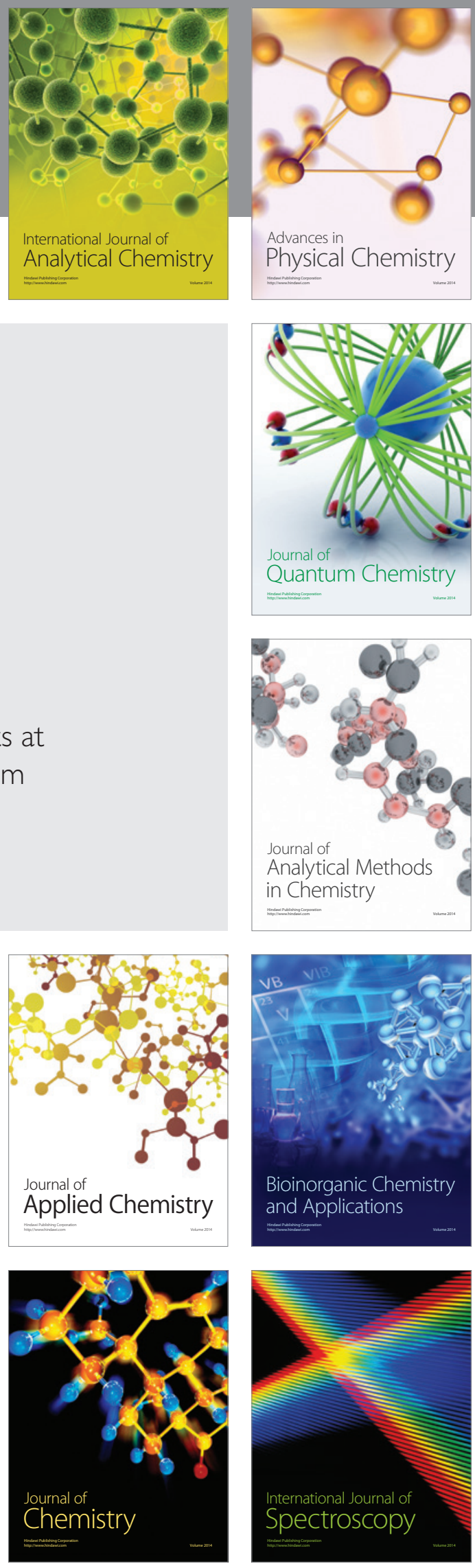